\title{
The LHCb Upgrade
}

\section{P. Campana*†}

Laboratori Nazionali di Frascati dell'I.N.F.N., Frascati (Italy)

E-mail: pierluigi.campana@lnf.infn.it

LHCb in its present configuration will collect approximately $10 \mathrm{fb}^{-1}$ of data in the next 5 years of LHC operation, at an average luminosity of $2 \cdot 10^{32} \mathrm{~cm}^{-2} \mathrm{~s}^{-1}$. In the near future, refined theoretical predictions and lattice calculations, as well as searches for New Physics in the Flavour Sector will require more data coming from decay of heavy quarks. Here we present the motivations for an upgrade of $\mathrm{LHCb}$ and the current ideas on how to deal with the experimental challenges. At a luminosity of $2 \cdot 10^{33} \mathrm{~cm}^{-2} \mathrm{~s}^{-1}$, the goal is to collect $100 \mathrm{fb}^{-1}$, with a large increase in the capabilities of the detector, in particular in the detection of hadronic channels.

Flavor Physics and CP Violation 2009,

May 27 - June 1, 2009,

Lake Placid, NY, USA

* Speaker.

† On behalf of the LHCb Collaboration. 


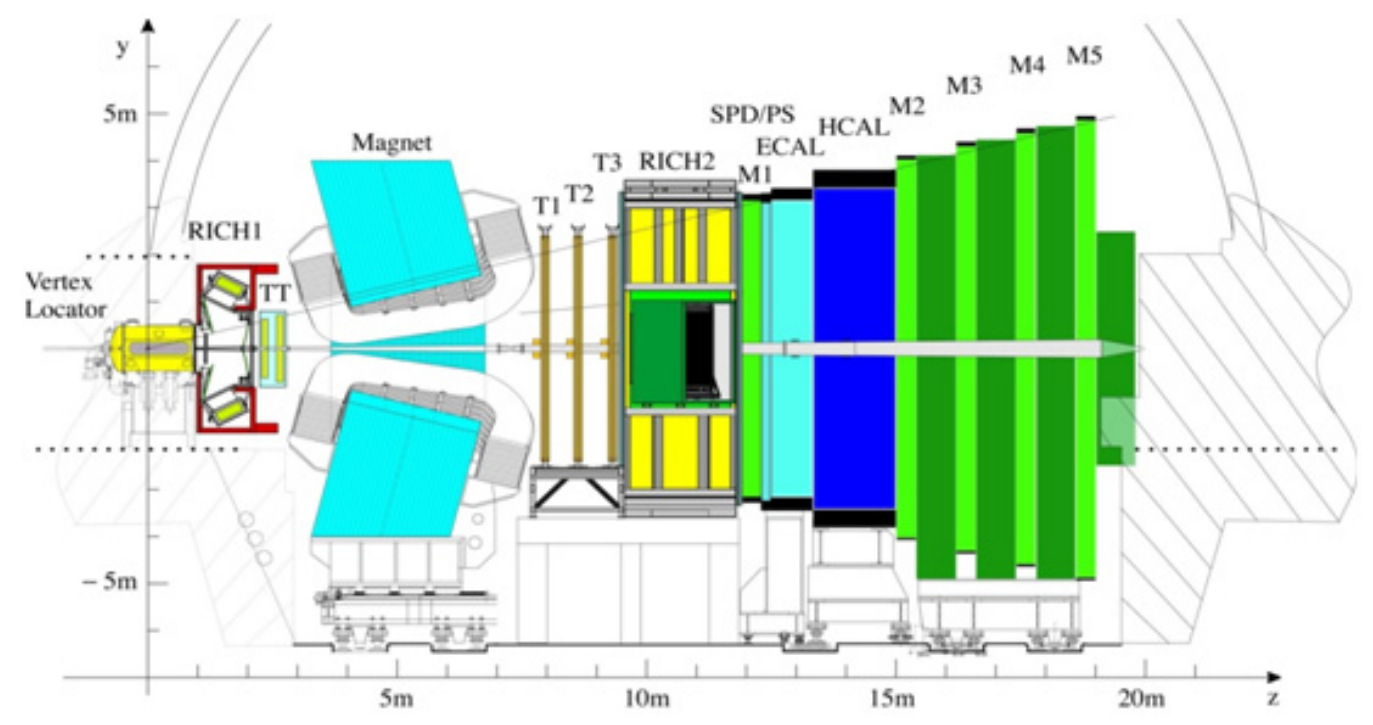

Figure 1: The LHCb detector.

\section{Introduction}

The LHCb experiment [1] is dedicated to study $\mathrm{CP}$ violation and possible deviations from Standard Model (SM) expectations in the Flavour Sector, particulary in the decay of B mesons. The detector is a one-arm spectrometer (Fig. 1) where both B mesons produced in pp collisions are detected, allowing for the exploitation of various techniques of B-tagging.

The operation at an hadron machine and the forward acceptance of the experiment, make the experimental conditions very tough: at $\sqrt{s}=14 \mathrm{TeV}$, it is expected to have a signal cross section ( $b \bar{b}$ events) of $\sigma_{b \bar{b}} \sim 0.5 \mathrm{mb}$ and an inelastic pp cross section approximately 160 times bigger. To reduce the strong background of minimum bias events, the detector has been conceived to have excellent performances: a resolution in the impact parameter of $\sim 30 \mu \mathrm{m}$; a resolution in the proper time of $\sim 40 \mathrm{fs}$; a resolution in the $B_{s}$ invariant mass of $\sim 14 \mathrm{MeV}$.

The LHCb experiment benefits also of several other relevant characteristics: the identification of hadrons in the RICH, to tag and reconstruct B meson final states; the first level trigger (L0) on high transverse energy and momentum $\left(E_{T}, p_{T}\right)$ leptons, photons and hadrons, to reduce the rate from $10 \mathrm{MHz}$ to approximately $1 \mathrm{MHz}$, which is the maximum tolerable rate in the present $\mathrm{LHCb}$ readout; an efficient high level software trigger (HLT), to produce output streams totaling no more than $2 \mathrm{kHz}$ useful for physics analysis. The experiment will operate at a luminosity in the interval $2 \div 5 \cdot 10^{32} \mathrm{~cm}^{-2} \mathrm{~s}^{-1}$ (a factor $50 \div 20$ below the LHC nominal one).

The machine optics of LHCb allows to run the experiment at such a luminosity, and even at least at a factor 10 above. Therefore a possible upgrade in luminosity of LHCb does not imply the increase of LHC luminosity.

Here we present and discuss the physics program of $\mathrm{LHCb}$, its trigger performances in the present layout and the motivations for an upgrade, together with the current ideas on how to overcome the limitations in $\mathrm{LHCb}$ to exploit efficiently an increase in luminosity. 


\section{LHCb and its physics programme}

In the next 5 years, LHCb will collect approximately $10 \mathrm{fb}^{-1}$ of data to look for signatures of New Physics (NP) in the Flavour Sector. We summarize the main highlights of the LHCb physics program in Tab. 1 (for a complete overview of the LHCb physics reach, see [2]).

LHCb is expected to start its data taking by the end of 2009 , and already with $200-300 p b^{-1}$ it will be competitive with Tevatron data in some areas (e.g. $B_{s} \rightarrow \mu \mu$ limit). A detailed description of the $\mathrm{LHCb}$ techniques and the sensitivities to the various physics parameters can be found in another talk at this conference [3].

Table 1: Highlights of the LHCb physics program with $10 \mathrm{fb}^{-1}$

\begin{tabular}{|c|r|}
\hline BR $\left(B_{s} \rightarrow \mu \mu\right)$ & $\sim 15 \%$ of SM value \\
Mixing phase in $B_{s} \rightarrow \psi \phi$ & $\sim 0.01$ \\
Mixing phase in $B_{s} \rightarrow \phi \phi$ & $\sim 0.05$ \\
Determination of $\gamma$ & $\sim 2.5^{\circ}$ \\
Zero of $A_{F B}(s)$ in $B_{d} \rightarrow K^{*} \mu \mu$ & $\sim 7 \%$ \\
\hline
\end{tabular}

\section{An upgrade in luminosity for $\mathrm{LHCb}$}

The LHCb Collaboration is considering a substantial upgrade of the detector, to run it at a luminosity of $2 \cdot 10^{33} \mathrm{~cm}^{-2} \mathrm{~s}^{-1}$ and to collect a factor $\mathrm{x} 20$ in statistics in hadronic channels and $\mathrm{x} 10$ in leptonic modes by the early years of next decade. The rationale for the upgrade is based on the goal of uncovering new physics effects in heavy flavor decays (both beauty and charm).

The present detector configuration of $\mathrm{LHCb}$ is optimized to maximize the signal over background ratio for the various streams of L0 first level trigger. The maximum rate tolerable by the
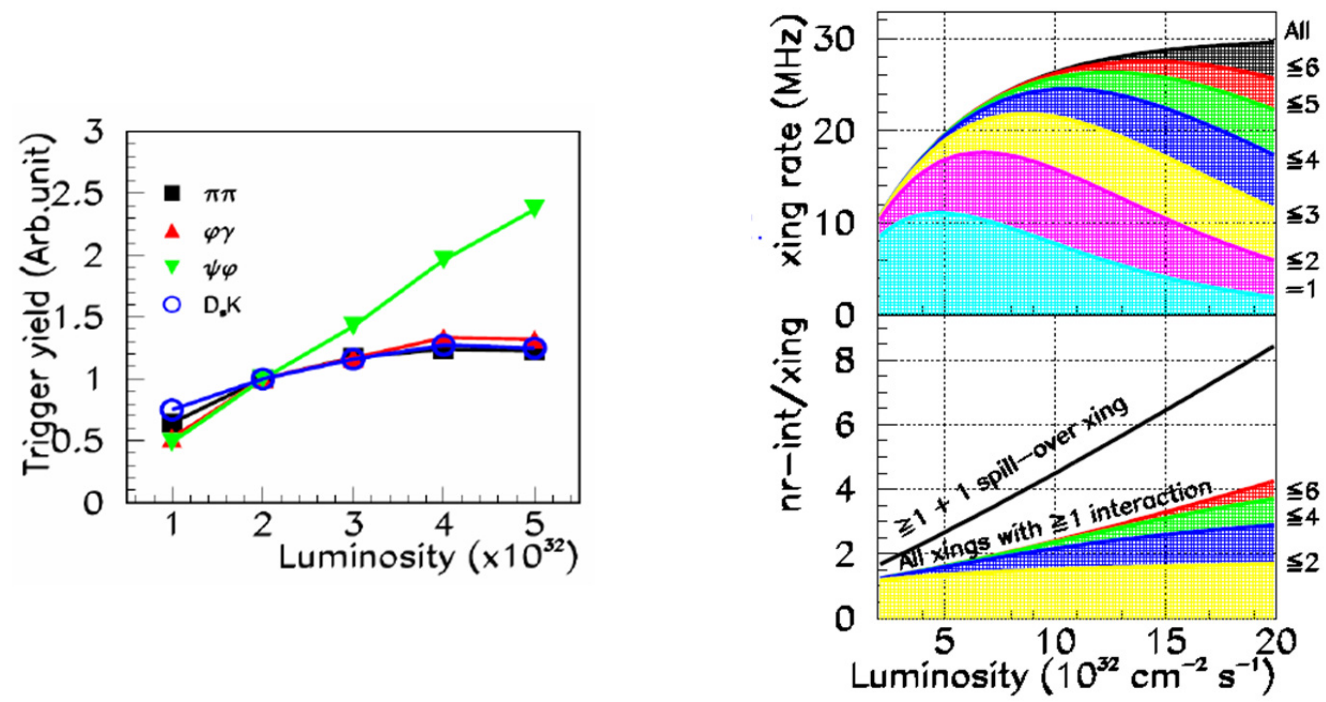

Figure 2: The trigger yield for various channels (left) and the multiplicities per interaction as a function of luminosity (right). 
electronic readout is set to $1 \mathrm{MHz}$, and the bandwidth is shared accordingly: $700 \mathrm{kHz}$ for the hadron trigger (with $E_{T}>3.5 \mathrm{GeV}$ ), $150 \mathrm{kHz}$ for the electron/photon trigger (with $p_{T}>2.5 \mathrm{GeV}$ ), and $150 \mathrm{kHz}$ for the muon trigger (with $p_{T}>1 \mathrm{GeV}$ ). A subsequent High Trigger Level (HLT1) confirms the L0 candidates (using partially the tracking and optionally the information on the impact parameter), and reduces the rate to $40 \mathrm{kHz}$; in a second step (HLT2) more refined exclusive and/or inclusive selections are performed bringing to a more limited sample to be stored on tape ( $2 \mathrm{kHz}$ ). The L0 trigger efficiency on hadronic channels is $\sim 40 \%$ (essentially due to the high $E_{T}$ threshold), while it is $\sim 90 \%$ on muon channels.

A luminosity upgrade keeping the current LHCb architecture would decrease the overall efficiency on hadronic events (see Fig. 2, left), both for the limit in bandwidth (one would be obliged to rise the thresholds) and for the increased complexity of events topology (many superimposed vertices). On the muon trigger side, yield would scale linearly with luminosity. The current baseline figure for the upgrade is $2 \cdot 10^{33} \mathrm{~cm}^{-2} \mathrm{~s}^{-1}$, a factor 5 still below the LHC nominal one.

The main consequences on data taking can be summarized as follows: $\sim 30 \mathrm{MHz}$ of crossing with at least one interaction (currently is $10 \mathrm{MHz}$ ); an average number of visible interactions per crossing of $\sim 5$ (currently is $\sim 2$ ); a spillover (hits coming from previous bunches) rising linearly with the luminosity (see Fig. 2, right). The LHCb strategy for the upgrade foresees to install on all subdetectors a FEE electronics capable of transferring data synchronously with the LHC clock (40 MHz). These data will be routed to large scale, fully software trigger farm which will be able to process, at first level, not only the calorimetric and muon high $p_{\Gamma}$ candidates, but also the information coming from the vertex and the tracking stations. This scheme will allow to rise the trigger efficiency on hadronic channels by a factor 2 , and to increase the total statistics in this class of events by a factor 20 . The goal is to collect, in 5 years at $2 \cdot 10^{33} \mathrm{~cm}^{-2} \mathrm{~s}^{-1}, 100 \mathrm{fb}^{-1}$. The upgrade to a $40 \mathrm{MHz}$ FEE readout has important implications on the subdetectors, as it will be discussed in the next paragraphs. In 2008, the LHCb Collaboration has submitted to LHCC an Expression of Interest for the Upgrade [4], and it is planning to prepare a TDR in 2010.

\section{New Physics discovery reach at $100 \mathrm{fb}^{-1}$}

It is well known that precision physics is an alternative tool for the search of deviations from the SM with respect to the one represented by the high energy frontier. The future prospects for this kind of searches at LHC can be summarized in the three following scenarios: LHC experiments might find hints or evidence for physics beyond the SM; Atlas and CMS might discover evidence for physics beyond the SM, but this effect is not observable within the present LHCb reach, suggesting that the Flavour Sector is protected against NP (as in MFV models [6]); detectors at LHC do not observe new effects.

All three scenarios are intriguing from the point of view of basic research knowledge, especially the second one, where a more refined search in rare or precision physics would elucidate the flavour structure of NP. It is also evident the difficulty of explaining the need for further investments in High Energy Physics in the third setting. In parallel with experimental increased precision, theoretical progresses in B physics, as well as refined lattice calculations, will ask for more data. Therefore the LHCb Collaboration is planning to upgrade the detector in a time scale of 6-7 years, so to match future long shutdown of the LHC machine (presently planned for 2015-16). 
The scale of the upgrade will depend heavily on the physics goals, on the available time and on the manpower and economic resources provided by the various funding agencies. In the following, we discuss few physics channels where a consistent increase in statistics could bring to significant new insight into the deviations from SM.

\section{1 $B_{d} \rightarrow K^{*} \mu \mu$}

This exclusive rare decay offers a rich phenomenology of various kinematical distributions beyond the measurement of the branching ratio (already performed at Belle). The main LHCb interest is in the study of some angular distribution: already with $10 \mathrm{fb}^{-1}$ one can measure the zeros of $A_{F B}(s)$. The large increase in statistics at an upgraded LHCb will make much higher precision measurements possible, beyond the forward-backward asymmetry, whose precision will be limited by theory. It has been recently pointed out [7] that with a very large statistics, several angular variables can be tested against NP, especially the so called transverse asymmetries, that are free from hadronic uncertainties in the controlled region $1<q^{2}<6 \mathrm{GeV}^{2}$ :

$$
A_{T}^{(2)}=\frac{\left|A_{\perp}\right|^{2}-\left|A_{\|}\right|^{2}}{\left|A_{\perp}\right|^{2}+\left|A_{\|}\right|^{2}} ; \quad A_{T}^{(3)}=\frac{\left|A_{0 L} A_{\| L}^{*}-A_{0 R}^{*} A_{\| R}\right|}{\sqrt{\left|A_{0}\right|^{2}\left|A_{\perp}\right|^{2}}} ; \quad A_{T}^{(4)}=\frac{\left|A_{0 L} A_{\perp L}^{*}-A_{0 R}^{*} A_{\perp R}\right|}{\left|A_{0 L}^{*} A_{\| L}+A_{0 R} A_{\| R}^{*}\right|}
$$

In $100 \mathrm{fb}^{-1} \mathrm{LHCb}$ it is expected to collect $\sim 350 \mathrm{k} B_{d} \rightarrow K^{*} \mu \mu$ events. The experimental determination of one of such a parameter is shown in Fig. 3.

\section{$4.2 B_{s} \rightarrow \phi \phi$}

Deviations from SM can be probed also by studying FCNCs in hadronic b-s transitions. One approach is to compare the time-dependent $\mathrm{CP}$ asymmetry in a hadronic penguin loop decay, where unknown massive particles could enter with a decay based on a tree diagram which generally is insensitive to NP and which has the same weak phase. In LHCb the best way is to compare mixing phases in the $B_{s} \rightarrow \psi \phi$ and $B_{s} \rightarrow \phi \phi$ decays. To accomplish this job, a fully angular analysis of the decay is needed, requiring a very high statistics. The SM prediction for $\beta_{s}^{e f f}$ is actually very close to zero [8], therefore any non zero value would indicate a clear signal of NP. The hadronic $B_{s} \rightarrow \phi \phi$ decay would particularly profit of the LHCb trigger upgrade as a first-level detached vertex trigger is required to reconstruct this decay mode. A yield of $\sim 300 \mathrm{k}$ events is expected in
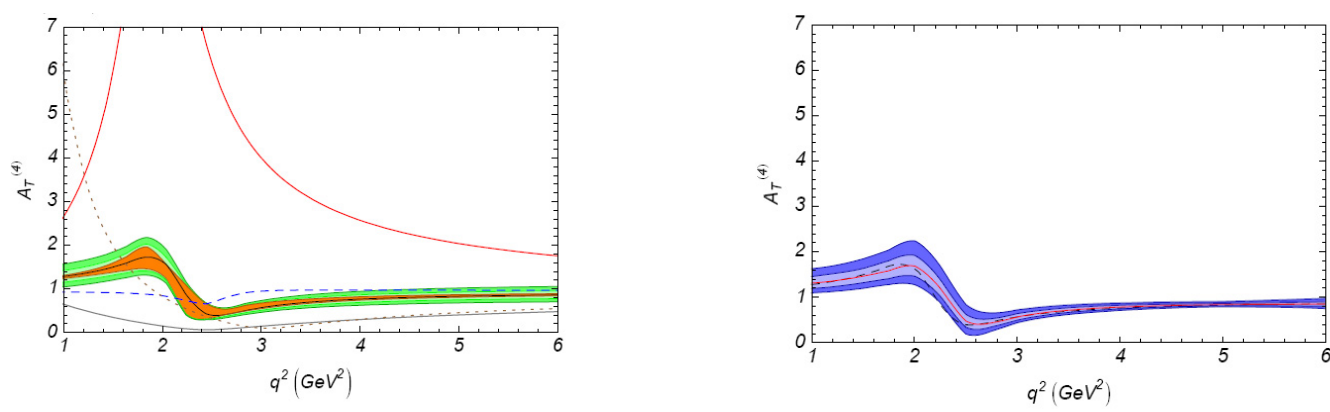

Figure 3: SM behaviour for the $A_{T}^{(4)}$ tranversity asymmetry (left, green band, together with predictions from several models, coloured lines) and its experimental determination with $100 \mathrm{fb}^{-1}$ (right, blue band). 
$100 \mathrm{fb}^{-1}$, and scaling the sensitivity we estimate a precision of 0.015 (ignoring for the moment any systematical error).

\subsection{Other channels}

The list of search opportunities at an upgraded LHCb is extensive, and we refer to the Expression of Interest for a deeper discussion. Here we mention few of them: the possibility of measuring the CKM angle $\gamma$ at a sub degree level, the observation of $B_{s} \rightarrow \mu \mu$ up to $10^{-9}$ level (of extreme relevance in many theoretical models [9]), the search for TDCPV in $B_{s} \rightarrow \phi \gamma$, the measurement of $\beta$ vs. $\beta^{e f f}$, the study of mixing and CPV in the charm sector, search for NP in $B_{s} \rightarrow \phi \mu \mu$, the observation of very rare decays $\left(B_{d} \rightarrow \mu \mu, \tau \rightarrow \mu \mu \mu\right)$.

\section{The experimental challenges of an upgraded LHCb}

\subsection{Vertexing}

Vertexing in high track density environment, as the one expected at an upgraded LHCb is by far one of the most relevant issue to be addressed. New running conditions of $\mathrm{LHCb}$ will require technical solutions more radiation hard and with improved pattern recognition [10]. Particularly challenging is the task of resolving several independent events (with detached vertices) in the same collision, in a beam envelope that has a longitudinal spread of $\sigma \sim 5 \mathrm{~cm}$.

Currently, the LHCb VELO group is considering three possible detector configurations. One has a layout similar to the present one, with shorter strips ("striplets") to decrease the ghost track rate: the active elements have only $6 \mathrm{~mm}$ long strips with very short routing lines, to beat down noise. The number of stations is similar to the present one (21). Thin sensors $(200 \mu \mathrm{m})$, cooled and stiffened by diamond spine, would guarantee a minimal amount of material. This solution has several challenges (mechanics, cooling, readout) to be faced. A first pixel option is based on

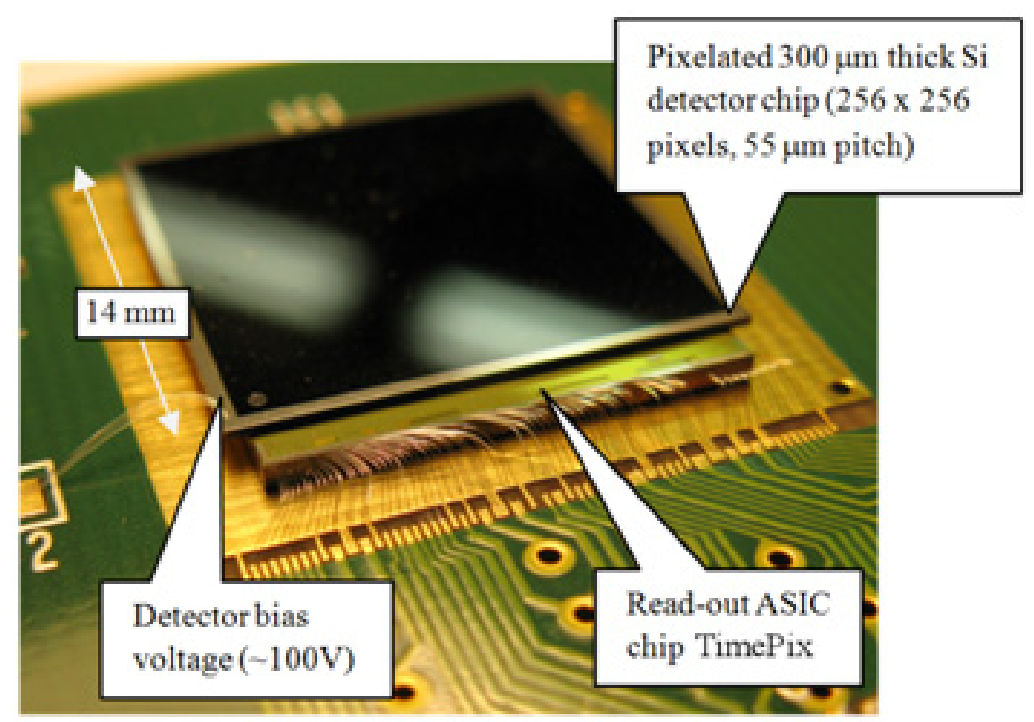

Figure 4: The TIMEPIX pixel chip. 
the FPIX chip (the active element having an area of 50x400 $\mu \mathrm{m}^{2}$ ) developed by BTEV design, each station with a double layer of staggered sensors, so to minimize dead regions. This solution appears, at the moment, the one with the highest material budget. Another pixel option makes use of the TIMEPIX chip (the active element having an area of 55x55 $\mathrm{m}^{2}$ ) (Fig. 4) and benefits of the extremely small dead region of the MEDPIX2 [11] chip, allowing for stations with a single sensor. However, the present imaging chip has a very slow readout. An upgrade of the chip to the $90 \mathrm{~nm}$ technology is foreseen.

An intense $\mathrm{R} \& \mathrm{D}$ is needed to address several problems: the radiation hardness (the expected fluence in $100 \mathrm{fb}^{-1}$ is $\sim 1.4 \cdot 10^{16} n_{e q} \mathrm{~cm}^{-2}$ ); the requirement of thinning the sensors, so to have the minimal amount of material; the search for new cooling solutions; the possible removal of the $\mathrm{RF}$ foil that accounts for half of the budget material; the request of positioning the detectors at a smaller radius.

\subsection{Tracking}

To assign momentum to VELO objects, the tracking in LHCb is performed in a mixed silicongas system: at low angles, with high granularity silicon devices (Inner Tracker); at higher angles, where occupancy is less severe, with straw tubes (Outer Tracker), $5 \mathrm{~mm}$ in diameter and up to 50 ns drift time. Preliminary results from simulation show that at $2 \cdot 10^{33} \mathrm{~cm}^{-2} \mathrm{~s}^{-1}$ occupancy in OT increases up to $25 \%$ (Fig. 5), with a large contribution due to spillover events. On top of this, the long projectivity of the OT increases the ghost track rate all over the active area. The request of having a $40 \mathrm{MHz}$ readout for the tracking system, forces to rebuild the whole FEE both for the gas and the silicon devices. Moreover, a change in the FEE of the Inner Tracker would imply a complete redesign of the sensors. The increase in occupancy suggests also to enlarge the area at small angle, to cover it with high granularity detectors (at least a factor of 4 in surface).

While silicon and straw tubes are still a likely choice for the tracking system, these considerations also suggested a change of technology for the upgrade of the tracking system: a brand

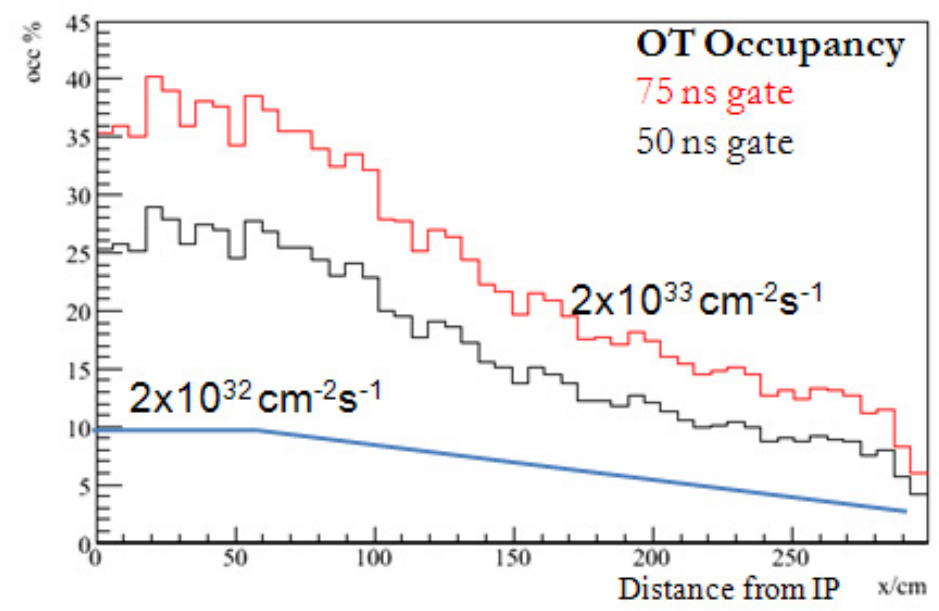

Figure 5: LHCb Outer Tracker occupancy as a function of distance from Interaction Point. 
new detector based on scintillating fibres. A preliminary layout foresees to have a large area fibre tracker made of mixed fibre dimensions ( $250 \mu \mathrm{m}$ in the inner region and $700 \mu \mathrm{m}$ in the outer), to be readout with SiPM and/or conventional Multi Anode PM. This solution would be compact and uniform with very little amount of services in the active zone, extremely good transversal granularity and excellent timing performances. The R\&D on the inner part already started with encouraging results: bundles of thin fibres, readout by dedicated SiPM arrays show a spatial resolution well within the one needed by LHCb [12] . Several issues have to be still addressed, such as fibre radiation hardness, optimisation of the photon readout and compatibility of the proposed layout with the present mechanical structure.

\subsection{Identification of hadrons}

The baseline approach for the upgrade of the $\mathrm{LHCb} \mathrm{RICH}$ counters is to mantain the present geometrical and mechanical layout. The need for a $40 \mathrm{MHz}$ FEE readout has the consequence that the photon detectors of the LHCb RICH have to be removed and substituted, as the electronics is bump bonded onto them. There are two possible options for the photosensors: new HPD or flat panel PM. The first option would imply an intense R\&D to study the ageing due to ion feedback increase and a search for new industrial solutions. On the other end, flat panel PM have the big appeal of being a mature product, available on the shelf. The required granularity is still in the range of $1 \times 1 \mathrm{~mm}^{2}$. Moreover, there is a proposal of replacing the Aerogel (and therefore saving some material budget) with a new TOF system, located after RICH2, in front of the calorimeters, to identify kaons in the momentum range below $10 \mathrm{GeV}$.

The plan of such a detector [13] is to install large quartz plates $(1 \mathrm{~cm}$ thick) at the entrance

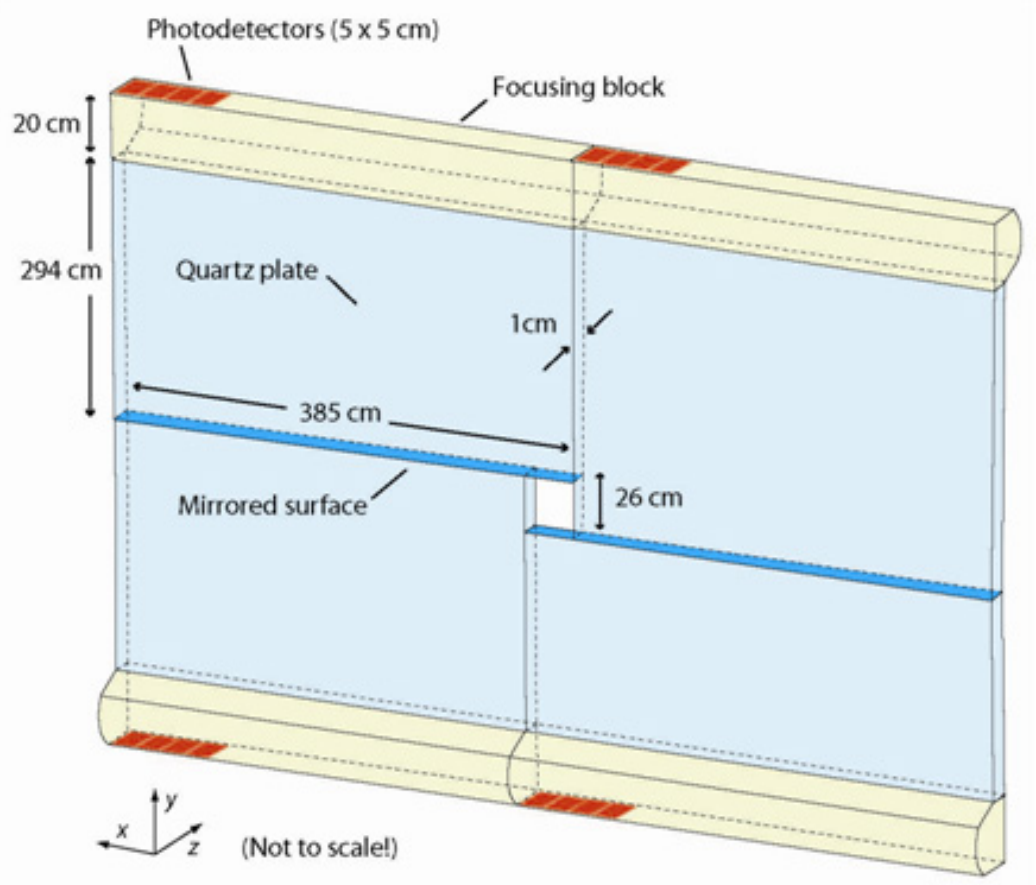

Figure 6: Concept design for a quartz-based TOF system in LHCb. 
of the calorimeters, and to measure TOF of impinging particles with a resolution in the range of 40 ps, using Micro Channel Plate PM (MCP) with multianode readout. A special focusing system is needed to measure $\theta_{\text {Cherenkov }}$ so to correct for the chromatic dispersion of the material and get the correct time of arrival of the photons. Recent data at test beams [14] have shown that MCP can achieve extremely good time resolution (10-20 ps) even in the presence of a limited amount of photelectrons (30-20 p.e.). The R\&D on several pending issues has just started, such as the feasibility of large quartz plates, the ageing of MCP, and the readout electronics.

\subsection{Calorimetry and Muon Systems}

In LHCb Calorimeters the FEE is already readout at $40 \mathrm{MHz}$, and therefore the changes to the electronics can be considered of limited amount. One major problem is represented by the accumulated dose in the inner zone of the calorimeter: scintillator modules have been tested up to $2.5 \mathrm{Mrad}$, while at high luminosity a larger dose (up to factor 5) is expected. Currently the Calorimeter group is planning to replace the hottest modules with $\mathrm{PbWO}$, although even in this case $R \& D$ on radiaton hardness is needed. It is also foreseen to reduce the PM gain (to limit the ageing) with a corresponding upgrade in preamp sensitivity to lower the noise. Pile-up effects at high luminosity are also under study. At $2 \cdot 10^{33} \mathrm{~cm}^{-2} \mathrm{~s}^{-1}$ energy resolution will be dominated at small angle by pileup contribution, therefore preventing from doing low $p_{\Gamma}$ gamma physics.

The Muon system FEE is readout at $40 \mathrm{MHz}$ too, and the plan is to keep the present electronic boards. At high luminosity there are two main concerns for the inner regions: the dead time induced by the high rate, and the ageing due to the radiation dose. In particular, it is expected that M1, in front of the calorimeters, will be removed due to high occupancy. A real evaluation of these two phenomena will come only from the first data, as the simulation of the background has a very large uncertainty. In case of too high rate and/or dose, the Muon group is considering the replacement with GEM (present now only on M1 station) and a reduction of the detector granularity, to match each physical channel with the rate limitation of the present front end (fully efficient only below 1 $\mathrm{MHz})$.

\subsection{Trigger and DAQ}

The realization of a trigger farm, based on commercial commodities, able to crunch the enormous amount of data coming at $40 \mathrm{MHz}$ from LHCb subdetectors, is one of the most challenging aspect of the upgrade. From several MonteCarlo studies, it is evident that at high luminosity the L0 trigger must reconstruct all primary vertices per bunch crossing, measure the impact parameters

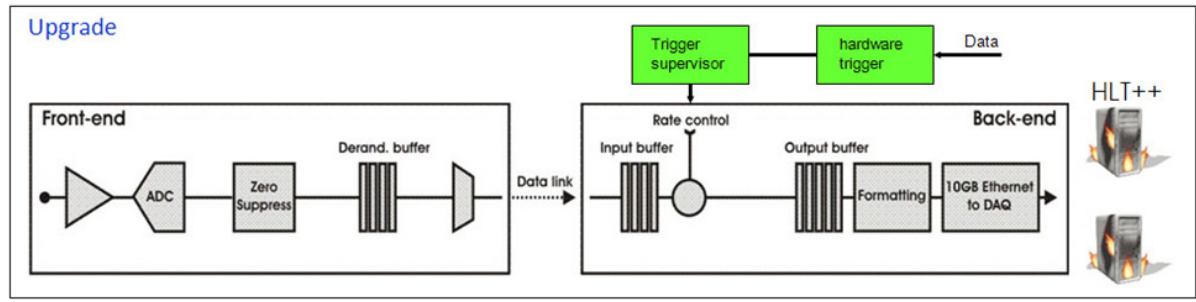

Figure 7: The readout configuration for an upgraded $\mathrm{LHCb}$ detector. 
of tracks belonging to any primary vertex, and measure the $p_{T}$ of tracks with significant impact parameter.

The current plan foresees to setup a trigger farm, with adequate buffer and bandwidth to perform selection cuts simultaneously on high $p_{T}$ and high impact parameter tracks. The possibility to reduce the input rate to the trigger farm using hardware external trigger (very similar to the present L0 Calo and Muon triggers) is also under consideration, together with the development of more efficient selection criteria and fast pattern recognition algorithms. Without the combination of all these elements the upgrade would not be enough to reach the required sensitivity.

Preliminary studies of this trigger scheme on $B_{s} \rightarrow \phi \phi$ events at a luminosity of $210^{33} \mathrm{~cm}^{-2} \mathrm{~s}^{-1}$ show an increase in efficiency of about a factor 2 , keeping the minimum bias rate at a reasonable level. A trigger strategy that executes in the requisite time and produces the required increase in efficiency for hadronic modes is under investigation. A sketch of the DAQ configuration for the LHCb upgrade is shown in fig. 7, while a detailed description of the DAQ layout can be found in another talk at this conference [15].

\section{Conclusions}

The LHCb experiment is ready at PIT 8 and waiting for LHC first data, and the plan is to collect $10 \mathrm{fb}^{-1}$ in 5 years. A substantial upgrade of LHCb in 2015-16 is under study, to run the detector at a luminosity of $2 \cdot 10^{33} \mathrm{~cm}^{-2} \mathrm{~s}^{-1}$ and to collect a factor $\mathrm{x} 20$ in statistics in hadronic channels and $\mathrm{x} 10$ in leptonic modes by the early years of next decade.

This statistics, supported by a substantial decrease of theoretical uncertainties and a deeper understanding of the detector systematics, could bring the Flavour Sector to an unprecedented degree of precision. This approach is largely complementary to the physics program of the proposed Super Flavor Factories.

We have discussed the present $\mathrm{LHCb}$ limitations and the challenges to be faced to overcome these difficulties. The Collaboration is starting actively to work on the ideas reported here. The modifications to be done to the $\mathrm{LHCb}$ detector are relevant, in particular those foreseen to upgrade the performances of the vertexing and of the tracking systems and to setup of the new triggering scheme at $40 \mathrm{MHz}$.

Nevertheless we think that, provided the adequate level of funding and manpower, the LHCb upgrade could become a reality in the near future.

\section{Acknowledgements}

This talk would not have been possible without the work done by many colleagues of LHCb, especially those contributing to the "LHCb Upgrade Working Group". I would like to thank in particular Paula Collins for providing material on vertex detectors, Hans Dijkstra for several useful discussions, Roger Forty and Sheldon Stone for a careful reading of the manuscript. 


\section{References}

[1] The LHCb Collaboration, LHCb Reoptimized Detector Design and Performance, CERN/LHCC 2003-030; The LHCb Detector at LHC, JINST 3 (2008) S08005.

[2] B, D and $K$ decays, Report of Working Group 2 of the CERN Workshop "Flavour in the era of LHC", Geneva (Switzerland), arXiv:0801.1833.

[3] O. Dechamps, Flavor physics techniques and sensitivities at LHCb, these Proceedings.

[4] The LHCb Collaboration, Expression of Interest for an LHCb Upgrade, CERN/LHCC 2008-007.

[5] H. Dijkstra, arXiv:0708:2665.

[6] G. Isidori, arXiv:0801:3039.

[7] U. Egede et al., JHEP 11 (2008) 32.

[8] M. Raidal, Phys. Rev. Lett. 89 (2002) 231803.

[9] J. Ellis et al., JHEP 10 (2007) 92; W. Altmannshofer et al., JHEP 1 (2009) 19; A. Dedes et al., Phys. Rev. D 79 (2009) 055006.

[10] M.Artuso, arXiv:0903.1605.

[11] For an description of the TIMEPIX chip, see http://medipix.web.cern.ch/MEDIPIX/index.html .

[12] G. Roper Yearwood et al., arXiv:0812.0454

[13] R. Forty, The LHCb RICH system, talk given at TIPP09, Tsukuba (Japan), March 11-17, 2009 (http://kds.kek.jp/contributionDisplay.py?contribId=45\&sessionId=33\& confId=2376)

[14] E. Ramberg, Test of 10 ps TOF Ph.Det. in FNAL test beam, talk given at TIPP09, (http://kds.kek.jp/contributionDisplay.py?contribId=52\&sessionId=42\& confId=2376)

[15] B. Jost, DAQ systems and technologies for Flavor Physics, these Proceedings. 